\title{
Organotypic tissue culture investigation of homocysteine thiolactone cardiotoxic effect
}

\author{
EV Lopatina ${ }^{1,2}$, AV Kipenko ${ }^{1,2}$, VA Penniyaynen ${ }^{1}$, NA Pasatetskaya ${ }^{1,2}$, \\ D Djuric ${ }^{3}$, BV Krylov ${ }^{1}$ \\ ${ }^{1}$ Pavlov Institute of Physiology Russian Academy of Sciences, St. Petersburg, Russia \\ ${ }^{2}$ Almazov Federal Heart, Blood and Endocrinology Centre, St. Petersburg, Russia \\ ${ }^{3}$ Institute of Medical Physiology "Richard Burian" Faculty of Medicine, University of Belgrade, \\ Belgrade, Serbia
}

Received: December 11, 2014

Accepted after revision: February 24, 2015

\begin{abstract}
Homocysteine thiolactone was demonstrated to inhibit the growth of 10-12-day-old chicken embryo cardiac tissue explants at $7 \times 10^{-9}-1 \times 10^{-3} \mathrm{M}$ concentrations in a dose-dependent manner. The maximal cardiotoxic effect of homocysteine thiolactone was detected at $1 \times 10^{-3} \mathrm{M}$, which corresponds to severe hyperhomocysteinemia. The results of experiments on culturing of cardiac tissue explants in the medium containing homocysteine thiolactone $\left(1 \times 10^{-3} \mathrm{M}\right)$ and ouabain at concentrations regulating the signal-transducing $\left(1 \times 10^{-10} \mathrm{M}\right)$ and pumping $\left(1 \times 10^{-8} \mathrm{M}\right)$ functions of $\mathrm{Na}^{+}, \mathrm{K}^{+}$-ATPase indicate that the cardiotoxic effect of homocysteine thiolactone is supposed to result from inhibition of the $\mathrm{Na}^{+}, \mathrm{K}^{+}$-ATPase pumping function.
\end{abstract}

Keywords: organotypic culture, cardiac tissue explants, PlasDIC-contrast, homocysteine thiolactone, $\mathrm{Na}^{+}, \mathrm{K}^{+}$ATPase.

The changes in homocysteine and homocysteine thiolactone blood level can be regarded as markers of developing cardiovascular or neurological pathology. An increase in blood concentrations of these agents is especially harmful for pregnant women, as homocysteine produces a pronounced neurotoxic effect during the fetal life $(1,7,9)$. Cardiac remodeling, which is characterized by accumulation of interstitial and perivascular collagen, was detected in hyperhomocysteinemic animals (2). The data about mechanisms of cardiac remodeling upon homocysteine action are controversial $(11,13)$.

The influence of homocysteine thiolactone on cell growth and proliferation in 10-12day old chicken embryo cardiac tissue explants is studied in the present paper by organotypic tissue culture method.

\section{Materials and Methods}

\section{Culture medium}

pH 7.4: 45\% - Hank's solution; 40\% - Eagle's medium; 15\% - fetal cow serum; supplemented with glucose $(0.6 \%)$, insulin $(0.5 \mathrm{ED} / \mathrm{ml})$, gentomycin $(100 \mathrm{ED} / \mathrm{ml})$, glutamine $(0.35 \%)(6)$.

Corresponding author: Ekaterina V. Lopatina, Sci. Dr., Ph.D.

Pavlov Institute of Physiology RAS

Makarova emb., 6 199034, St. Petersburg, Russia

Phone: +7(812)3281301; Fax: +7(812)3280501; E-mail: evlopatina@yandex.ru 
Drugs

Homocysteine thiolactone (Sigma, USA) and ouabain (Sigma, USA) were added to the experimental Petri dishes. Control explants were cultured in standard culture medium.

\section{Animal preparation}

Experimental animals were 10-12-day-old White Leghorn chicken embryos provided by Sinyavino Poultry, Leningrad District, Russia. Cardiac tissue explants were obtained by extirpation from the ventricle of heart.

\section{Organotypic tissue culture}

Studies were performed on 800 explants. Cardiac tissue explants were cultured for three days on collagen supports in Petri dishes at $37{ }^{\circ} \mathrm{C}$ with $5 \% \mathrm{CO}_{2} . \mathrm{CO}_{2}$-incubator (Sanyo, Japan) was used. Each Petri dish contained approximately 25 explants.

The growth of explants in tissue culture was controlled on vital preparations using a confocal laser scanning microscope LSM-710 (Carl Zeiss, Germany). The preparations were also visualized by a television microscope attachment Axiostar Plus (Carl Zeiss, Germany). Acridine orange $(0.01 \%)$ was used to stain the explants.

\section{Microscopic research methods}

A laser confocal microscope (LSM-710, Carl Zeiss, Germany) was integrated with a standard Carl Zeiss Axio Observer Z1 inverted optical microscope. A PlasDIC-contrast was used for better visualization of organ culture growth in plastic Petri dishes.

\section{Hill coefficient calculation}

The cardiac tissue explants area index dependence on homocysteine thiolactone concentration was approximated by the Hill equation:

$$
\mathrm{AI}=1-1 /\left[1+\left(\mathrm{K}_{\mathrm{D}} /[\mathrm{HT}]\right)^{\mathrm{X}}\right],
$$

where $\mathrm{AI}$ is the area index value, $\mathrm{K}_{\mathrm{D}}$ - homocysteine thiolactone concentration inducing a $50 \%$ decrease in AI, [HT] - homocysteine thiolactone concentration, $\mathrm{X}$ - the Hill coefficient.

\section{Statistical analysis}

Cardiac tissue explants growth was quantified using the ImageJ program. The area index (AI) was used to estimate the explants growth. It was calculated in relative units as the ratio of total explant area to the central zone area. AI value was taken as $100 \%$ in the control experiments. The data were processed with STATISTICA 6.0 and Student's $t$-test at $p=0.05$.

\section{Results}

An intravital microphotograph of 10-day-old chicken embryo cardiac tissue explant made by a confocal laser scanning microscope LSM-710 (Carl Zeiss, Germany) using a PlasDICcontrast is presented in Fig. 1. In the course of vital microscopic examination two zones could be observed in the explants after three days of culturing: the central zone (more dense) and the less optically dense peripheral zone, which surrounds the central zone. In both control and experimental explants the peripheral zone (the growth zone) comprised mostly proliferating cardiomyocytes (multinucleate polyploid cross-striated spindle-shaped cells, 65-70\%), as well as fibroblasts (up to 35\%) (Fig. 2). In some experiments a spontaneous contractive activity of cardiomyocytes was observed and registered by real-time photography. 

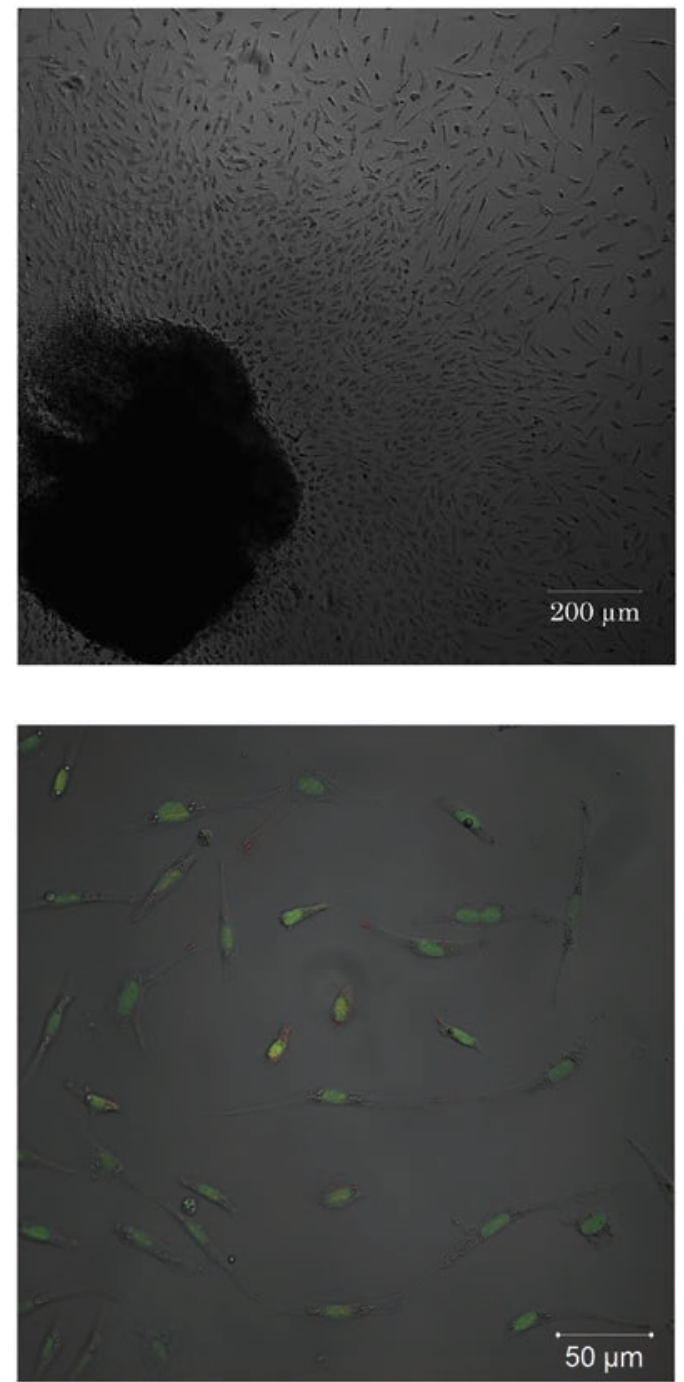

Fig. 1. Microphotograph of 10-day-old chicken embryo cardiac tissue explant. Control experiments. (3rd day of culturing, magnification 10, LSM-710, Carl Zeiss). PlasDIC-contrast

Fig. 2. Microphotograph: the growth area of 10-dayold chicken embryo cardiac tissue explant. Control experiments. (3rd day of culturing, magnification 40, LSM-710, Carl Zeiss) After staining with acridine orange

Influence of homocysteine thiolactone on 10-12-day-old chicken embryo cardiac tissue explants growth was investigated at $7 \times 10^{-9}-1 \times 10^{-3} \mathrm{M}$ agent concentrations (Fig. 3). Upon addition of homocysteine thiolactone at $7 \times 10^{-9} \mathrm{M}$, a $13 \%$ decrease in AI of experimental explants was detected after three days of culturing. AI of experimental explants cultured in the medium containing homocysteine thiolactone at $4 \times 10^{-8} \mathrm{M}$ was $26 \%$ less than the control value. A statistically significant inhibition (33\% and $29 \%$, correspondingly) of explant growth was observed upon addition of homocysteine thiolactone at $7 \times 10^{-7}$ and $8 \times 10^{-6} \mathrm{M}$ (Fig. 3). At $1 \times 10^{-3} \mathrm{M}$ homocysteine thiolactone inhibited the growth of explants by a mean of $60 \%$, thus expressing a pronounced cardiotoxic effect. Homocysteine thiolactone did not exhibit any growth-stimulating effect on 10-12-day-old chicken embryo cardiac tissue explants.

The usage of a confocal microscope made it possible to perform a 3-D reconstruction of investigated explants, to optically register the formation of cell layers, to measure the 
thickness of the forming growth zone, and to compare the data obtained in the pharmacological analysis with the control data. A new criterion to estimate the explant growth was introduced - the thickness of the growth zone $(\mu \mathrm{m})$. This is the only approach to describe the synthetic processes occurring in the growth zone, which is formed layer-by-layer. It is necessary to note that so far the growth zone cells have been considered to form a monolayer (5). The thickness of the growth zone of cardiac tissue explants cultured in the medium with addition of homocysteine thiolactone did not differ from the control value at none of the tested concentrations of the agent and was equal to $22 \mu \mathrm{m}$.

Approximation of AI dependence on homocysteine thiolactone concentration by the Hill equation made it possible to estimate $\mathrm{K}_{\mathrm{D}}\left(10^{-4} \mathrm{M}\right)$. The Hill coefficient $(\mathrm{X})$ was equal to 0.18 , which might result from a negative cooperativity. Action of homocysteine thiolactone was monotonously dose-dependent. A cardiotoxic effect of homocysteine thiolactone was discovered. A statistically significant inhibition of cardiac tissue explants growth by homocysteine thiolactone was observed at $4 \times 10^{-8}-1 \times 10^{-3} \mathrm{M}$ concentrations of the agent.

In order to elucidate the putative mechanism of homocysteine thiolactone cardiotoxic action, a separate set of experiments was carried out. Cardiac tissue explants were cultured in the medium containing homocysteine thiolactone at its inhibiting concentration of $1 \times 10^{-3} \mathrm{M}$ together with ouabain at $1 \times 10^{-8} \mathrm{M}$ or $1 \times 10^{-10} \mathrm{M}$.

In accordance with our prior data, ouabain (a selective inhibitor of $\mathrm{Na}^{+}, \mathrm{K}^{+}$-ATPase, $1 \times$ $10^{-10} \mathrm{M}$ ) stimulates the growth of cardiac tissue explants by an average of $37 \%(6)$. It is the concentration of ouabain that regulates the signal-transducing function of $\mathrm{Na}^{+}, \mathrm{K}^{+}$-ATPase.

Ouabain being applied at $1 \times 10^{-8} \mathrm{M}$ inhibits the growth of cardiac tissue explants almost completely (6). AI value is equal to $5 \%$ of the control value. Ouabain at $1 \times 10^{-8} \mathrm{M}$ inhibits both the signal-transducing and pumping functions of $\mathrm{Na}^{+}, \mathrm{K}^{+}$-ATPase.

AI of experimental explants cultured in the medium containing homocysteine thiolactone at $1 \times 10^{-3} \mathrm{M}$ and ouabain at $1 \times 10^{-10} \mathrm{M}$ was equal to $57 \%$ of the control value (Fig. 4). There were now observed the significant differences between the effects of homocysteine thiolactone $\left(1 \times 10^{-3} \mathrm{M}\right)$ and homocysteine thiolactone $\left(1 \times 10^{-3} \mathrm{M}\right)$ in phone ouabain $\left(1 \times 10^{-10} \mathrm{M}\right)$. AI of the explants cultured in the presence of homocysteine thiolactone at $1 \times 10^{-3} \mathrm{M}$ and ouabain at $1 \times 10^{-8} \mathrm{M}$ was equal to that of the explants cultured in the medium containing only ouabain. Thus, it is likely that the cardiotoxic effect of homocysteine thiolactone results from inhibition of the $\mathrm{Na}^{+}, \mathrm{K}^{+}$-ATPase pumping function.

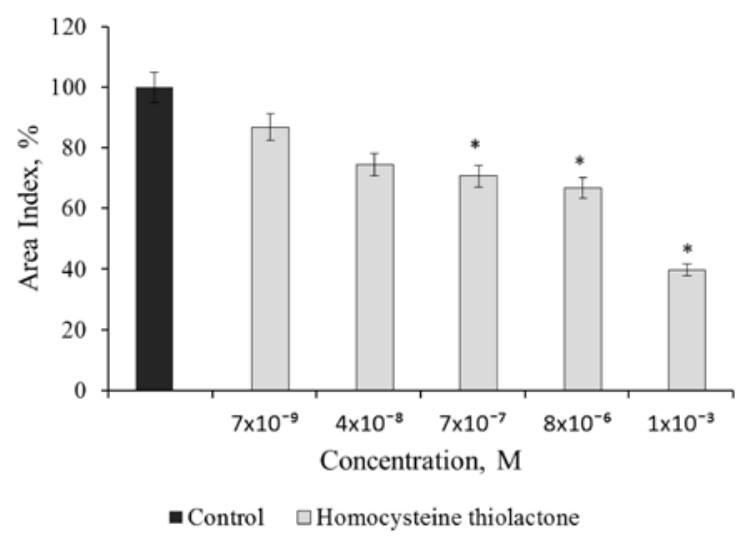

Fig. 3. Cardiotoxic effect of homocysteine thiolactone 


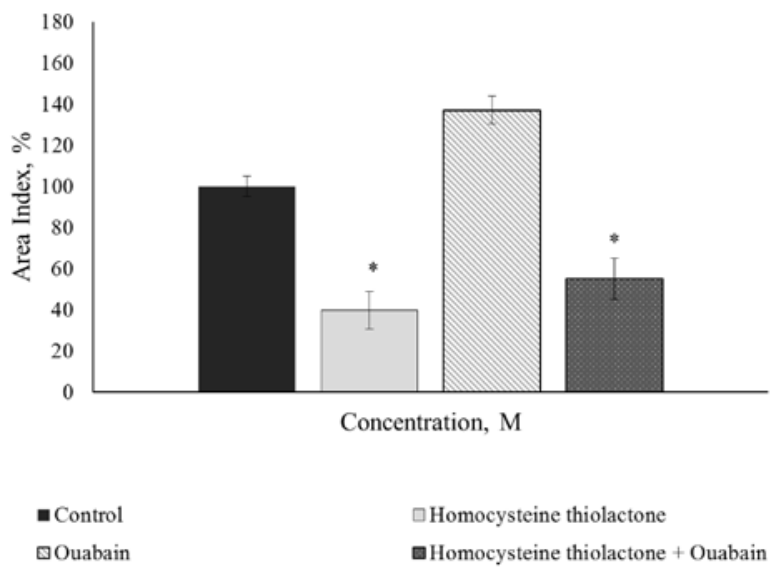

Fig. 4. Combined effect of homocysteine thiolactone $\left(1 \times 10^{-3} \mathrm{M}\right)$ and ouabain $\left(1 \times 10^{-10} \mathrm{M}\right)$ on 10-12-day-old chicken embryo cardiac tissue explants growth

\section{Discussion}

Apart from a negative effect on the cardiovascular system, an elevated concentration of homocysteine in the blood plasma provokes the development of neural tube defects (1), abnormal pregnancy (7) and neurodegenerative diseases (3). Hyperhomocysteinemia in humans also provokes, in particular, epileptic seizures (convulsions). Convulsive ( $8 \mathrm{mmol} / \mathrm{kg}$ ) and subconvulsive $(5.5 \mathrm{mmol} / \mathrm{kg})$ homocysteine dosage was used in the development of models for testing the activity of various anticonvulsive drugs (8).

Inhibition of $\mathrm{Na}^{+}, \mathrm{K}^{+}$-ATPase activity upon hyperhomocysteinemia was detected using the experimental epilepsy model $(3,12)$. A study on $\mathrm{Na}^{+}, \mathrm{K}^{+}$-ATPase kinetics has demonstrated that homocysteine inhibits the pumping function of the enzyme $(3,12)$. Homocysteine effect on the $\mathrm{Na}^{+}, \mathrm{K}^{+}$-ATPase signal-transducing function was not investigated.

Homocysteine thiolactone inhibits cardiac tissue explants growth in a dose-dependent manner, as demonstrated in the current paper. The maximal cardiotoxic effect of homocysteine thiolactone was achieved at $1 \times 10^{-3} \mathrm{M}$. This concentration corresponds to severe hyperhomocysteinemia. AI of experimental explants was $60 \%$ lower than the control value.

A putative reason for cardiac remodelling upon homocysteine action could be an increase in the number of mast cells which release mediators inducing cardiomyocytal hypertrophy, cell death or fibrosis $(4,10)$. Application of the organotypic tissue culture method made it possible to estimate the direct influence of homocysteine thiolactone on a cardiac tissue cell community. The responses of the immune system, which should be taken into consideration upon in vivo administration of the agent, were thus eliminated. Results of cardiac tissue explants culturing in the medium containing homocysteine thiolactone $\left(1 \times 10^{-3} \mathrm{M}\right)$ and ouabain at effective concentrations that regulate the signal-transducing $(1 \times$ $\left.10^{-10} \mathrm{M}\right)$ and pumping $\left(1 \times 10^{-8} \mathrm{M}\right)$ functions of $\mathrm{Na}^{+}, \mathrm{K}^{+}$-ATPase indicate that the cardiotoxic effect of homocysteine thiolactone presumably results from inhibition of the $\mathrm{Na}^{+}, \mathrm{K}^{+}$-ATPase pumping function. 


\section{Acknowledgements}

The experiments were carried out using the equipment of shared-use Center "Confocal microscopy" at Pavlov Institute of Physiology, Russian Academy of Sciences. Authors are grateful to Dr. I.V. Rogachevskiy (Pavlov Institute of Physiology, Russian Academy of Sciences) for valuable discussions. This project was supported by the Russian Scientific Foundation, research project №14-15-00677.

\section{REFERENCES}

1. Brauer PR, Tierney BJ: Consequences of elevated homocysteine during embryonic development and possible modes of action. Curr. Pharm. Des. 10, 2719-2732 (2004)

2. Herrmann W, Herrmann M, Joseph J, Tyagi SC: Homocysteine, brain natriuretic peptide and chronic heart failure: a critical review. Clin. Chem. Lab. Med. 45, 1633-1644 (2007)

3. Hrnčić D, Rasić-Marcović A, Krstić D, Macut D, Djuric D, Stanojlović O: The role of nitric oxide in homocysteine thiolactone-induced seizures in adult rats. Cell. Mol. Neurobiol. 30, 219-231 (2010)

4. Joseph J, Joseph L, Shekhawat NS, Devi S, Wang J, Melchert RB, Hauer-Jensen M, Kennedy RH: Hyperhomocysteinemia leads to pathological ventricular hypertrophy in normotensive rats. Am. J. Physiol. Heart Circ. Physiol. 285, H679-H686 (2003)

5. Konovalov YuB, Olenev EI, Chumasov YuA (1977): Tissue Culture of the Nervous System. «Medicine». Moscou. (in Russian Культура нервной ткани)

6. Lopatina EV, Penniyainen VA, Zaika AA: Regulatory role of Na,K-ATPase in the growth of heart tissue explants in organotypic culture. Bull. Exp. Biol. Med. 140, 184-186 (2005)

7. Perła-Kaján J, Twardowski T, Jakubowski H: Mechanisms of homocysteine toxicity in humans. Amino acids 32 , 561-572 (2007)

8. Rašić-Marković A, Stanojlović O, Hrnčić D: The activity of erythrocyte and brain $\mathrm{Na}^{+} / \mathrm{K}^{+}$and $\mathrm{Mg}^{2+}$-ATPases in rats subjected to acute homocysteine and homocysteine thiolactone administration. Mol. Cell. Biochem. 327, 39-45 (2009)

9. Rosenquist TH, Ratashak SA, Selhub J: Homocysteine induces congenital defects of the heart and neural tube: effect of folic acid. Proc. Natl Acad. Sci. USA 93, 15227-15232 (1996)

10. Sipkens JA, Krijnen PA, Meischl C, Cillessen SA, Smulders YM, Smith DE, Giroth CP, Spreeuwenberg MD, Musters RJ, Muller A, Jakobs C, Roos D, Stehouwer CD, Rauwerda JA, van Hinsbergh VW, Niessen HW: Homocysteine affects cardiomyocyte viability: concentration-dependent effects on reversible flip-flop, and necrosis. Apoptosis 12, 1407-1418 (2007)

11. Steed MM, Tyagi SC: Mechanisms of cardiovascular remodeling in hyperhomocysteinemia. Antioxid Redox Signal 15, 1927-1943 (2011)

12. Streck EL, Zugno AI, Tagliari B, Wannmacher CMD, Wajner M, Wyse ATS: Inhibition of Na+, K+-ATPase activity by the metabolites accumulating in homocystinuria. Metabolic Brain Disease 17, 83-91 (2002)

13. Zivkovic V, Jakovljevic V, Pechanova O, Srejovic I, Joksimovic J, Selakovic D, Barudzic N, Djuric DM: Effects of DL-homocysteine thiolactone on cardiac contractility, coronary flow, and oxidative stress markers in the isolated rat heart: the role of different gasotransmitters. Biomed. Res. Int. 2013, 318471 (2013) 